\title{
Effect of nicotine on orthodontic tooth movement and bone remodeling in rats
}

\author{
Sung-Hee Lee ${ }^{\mathrm{a}}$ (1) \\ Jung-Yul Cha ${ }^{\mathrm{b}}$ \\ Sung-Hwan Choi ${ }^{b}$ \\ Baek-il Kim ${ }^{\mathrm{c}}$ \\ Jae-Kook Cha ${ }^{\mathrm{d}}$ \\ Chung-Ju Hwang ${ }^{\mathrm{b}}$ (1)
}

${ }^{\mathrm{a}}$ Department of Orthodontics, College of Dentistry, Yonsei University, Seoul, Korea

${ }^{\mathrm{b}}$ Department of Orthodontics, Institute of Craniofacial Deformity, College of Dentistry, Yonsei University, Seoul, Korea

'Department of Preventive Dentistry of Public Oral Health, College of Dentistry, Yonsei University, Seoul, Korea

${ }^{\mathrm{d} D e p a r t m e n t}$ of Periodontics, College of Dentistry, Yonsei University, Seoul, Korea
Objective: To quantitatively analyze the effect of nicotine on orthodontic tooth movement (OTM) and bone remodeling in rats using micro-computed tomography and tartrate-resistant acid phosphatase immunostaining. Methods: Thirty-nine adult male Sprague-Dawley rats were randomized into three groups: group A, $0.5 \mathrm{~mL}$ normal saline ( $\mathrm{n}=9,3$ per 3, 7, and 14 days); group $\mathrm{B}, 0.83 \mathrm{mg} / \mathrm{kg}$ nicotine ( $\mathrm{n}=15,5$ per 3,7 , and 14 days); and group C, 1.67 $\mathrm{mg} / \mathrm{kg}$ nicotine ( $\mathrm{n}=15,5$ per 3,7 , and 14 days). Each animal received daily intraperitoneal injections of nicotine/saline from the day of insertion of identical 30-g orthodontic force delivery systems. A 5-mm nickel-titanium closed-coil spring was applied between the left maxillary first molar (M1) and the two splinted incisors. The rate of OTM and volumetric bone changes were measured using micro-computed tomography. Osteoclasts were counted on the mesial alveolar bone surface of the distobuccal root of M1. Six dependent outcome variables, including the intermolar distance, bone volume fraction, bone mineral density, trabecular thickness, trabecular volume, and osteoclast number, were summarized using simple descriptive statistics. Nonparametric Kruskal-Wallis tests were used to evaluate differences among groups at 3, 7, and 14 days of OTM. Results: All six dependent outcome variables showed no statistically significant among group-differences at 3, 7, and 14 days. Conclusions: The findings of this study suggest that nicotine does not affect OTM and bone remodeling, although fluctuations during the different stages of OTM in the nicotine groups should be elucidated in further prospective studies.

[Korean J Orthod 2021;51(4):282-292]

Key words: Bone remodeling, Micro-computed tomography, Nicotine, Orthodontic tooth movement

Received September 15, 2020; Revised November 23, 2020; Accepted January 20, 2021.

Corresponding author: Chung-Ju Hwang.

Professor, Department of Orthodontics, Institute of Craniofacial Deformity, College of Dentistry, Yonsei University, 50-1 Yonsei-ro, Seodaemun-gu, Seoul 03722, Korea.

Tel +82-2-2228-3106 e-mail hwang@yuhs.ac

How to cite this article: Lee SH, Cha JY, Choi SH, Kim Bl, Cha JK, Hwang CJ. Effect of nicotine on orthodontic tooth movement and bone remodeling in rats. Korean J Orthod 2021;51:282-292.

(C) 2021 The Korean Association of Orthodontists.

This is an Open Access article distributed under the terms of the Creative Commons Attribution Non-Commercial License (http://creativecommons.org/licenses/by-nc/4.0) which permits unrestricted non-commercial use, distribution, and reproduction in any medium, provided the original work is properly cited. 


\section{INTRODUCTION}

Nicotine has been reported to enhance constriction of the intact bone vasculature, impair angiogenesis and osteogenesis in areas of ossification, such as extraction sockets, and compromise wound healing following surgical therapy. 'It was also found to have a negative effect on bone remodeling via an increase in cytokines involved in bone resorption, ${ }^{2}$ and oxidative stress ${ }^{3,4}$ which induce osteoclastogenesis and inhibit osteoblastic differentiation. Nicotine itself has been observed to cause dose-dependent bone loss in furcation areas, even in rats with a healthy periodontium, and showed adverse effects on the dynamic histomorphometric parameters of the trabecular bone in a time-dependent manner. ${ }^{5-7}$

Orthodontic tooth movement (OTM) is mediated by coupling bone resorption and deposition on the compression and tension sides of the periodontal ligament, respectively. ${ }^{8}$ Tooth movement by orthodontic force application is characterized by remodeling changes in the dental and paradental tissues, including the dental pulp, periodontal ligament, alveolar bone, and gingiva. These force-induced strains alter vascularity and blood flow in the periodontal ligament, resulting in local synthesis and release of various key molecules such as neurotransmitters, cytokines, growth factors, colony-stimulating factors, and arachidonic acid metabolites. These molecules can affect bone remodeling and the rate of OTM. ${ }^{8}$ Many researchers have studied the effects of drugs, hormones, and systemic factors, ${ }^{9}$ growth factors, cytokines, prostaglandin, alveolar corticotomy causing the regional acceleratory phenomenon, ${ }^{10}$ and low-frequency mechanical vibration" on the rate of OTM and bone metabolism. Sodagar et al. ${ }^{12}$ and Bakathir et al. ${ }^{13}$ reported that nicotine significantly accelerated the OTM rate in a dosedependent manner in rats. However, both study groups measured the OTM rate by using an interproximal gauge only at 14 days. Moreover, Shintcovsk et al. ${ }^{14}$ documented that nicotine affected bone remodeling during OTM by reducing angiogenesis, osteoclast-like cells and Howship's lacunae, and delaying the collagen maturation process in the developed bone matrix.

With an increase in life expectancy and awareness of dental health and orthodontic treatment among adolescents, adults, and older adults, there is increased demand for esthetic and more satisfactory outcomes after comprehensive dental treatment using a multidisciplinary approach involving orthodontic treatment. Orthodontists should predict the desirable OTM rate, accompanying bone remodeling, and adaptive paradental tissue changes depending on the orthodontic force applied for mechanotherapy and the modulating factors such as systemic factors, drugs, oral hygiene, and smoking. To our knowledge, few studies have assessed the effect of nicotine on bone remodeling in a time- and dose-dependent manner in accordance with the stages of OTM.

Thus, the aim of this study was to quantitatively analyze the effect of nicotine on OTM and bone remodeling in rats using micro-computed tomography (microCT) and tartrate-resistant acid phosphatase (TRAP) immunostaining. We hypothesized that nicotine would deteriorate bone remodeling around teeth with optimal orthodontic forces and result in adverse consequences of OTM.

\section{MATERIALS AND METHODS}

\section{Experimental animals and study design}

The Institutional Animal Care and Use Committee of the University of Yonsei Health System (IACUC) approved this study (the approval number 2018-0252), which conformed to the Animal Research: Reporting of In Vivo Experiments (ARRIVE) guidelines. At the start of the experiment, 48 adult (9 weeks of age), male SpragueDawley rats (OrientBio, Seongnam, Korea) weighing 300 $\pm 50 \mathrm{~g}$ were kept in plastic cages under a standard 12hour light/dark photoperiod at a temperature of $21 \pm 2^{\circ} \mathrm{C}$ and humidity of $55 \%$. The rats were fed standard rat food and sufficient water ad libitum. The animals were allowed at least a week of acclimatization at the University of Yonsei Health System, and they were weighed at the beginning of the study and every week. On the basis of the literature, we determined the need for five experimental animals per group at each time point. ${ }^{6,15,16}$ The enrolled rats were randomly divided into three groups: group $\mathrm{A}$, control group, where rats received $0.5 \mathrm{~mL}$ normal saline; group B, where rats received $0.83 \mathrm{mg} / \mathrm{kg}$ nicotine (NOVO PREMIUM LIQUID $30 \mathrm{~mL}$, natural nicotine 0.95\%; NOVO, C\&L, Seongnam, Korea) dissolved in normal saline solution; and group $\mathrm{C}$, where rats received 1.67 $\mathrm{mg} / \mathrm{kg}$ nicotine (NOVO, C\&L). The two doses of nicotine, $0.83 \mathrm{mg} / \mathrm{kg}$ daily and $1.67 \mathrm{mg} / \mathrm{kg}$ daily, were representative of the doses ingested by humans who daily smoke 10 and 20 cigarettes containing $2.0 \mathrm{mg}$ of nicotine each, respectively. ${ }^{17}$ For insertion of the orthodontic appliance, the rats were anesthetized by intraperitoneal injection of a mixture of $0.9 \mathrm{mg} / \mathrm{kg}$ xylazine hydrochloride and 87 $\mathrm{mg} / \mathrm{kg}$ ketamine hydrochloride (YUHAN, Seoul, Korea) in the left groin area. Every animal received daily saline or nicotine injections from the day of insertion of identical $30-g$ orthodontic force delivery systems, and evaluations were performed at 3, 7, and 14 days (Figure 1).

\section{Orthodontic force application system}

The orthodontic force application system comprised a 5-mm nickel-titanium (Ni-Ti) closed-coil spring (MANCC10, $010 \times$ 030; Modern Arch, Wyomissing, PA, USA) 


\begin{tabular}{|c|c|c|}
\hline \multicolumn{2}{|c|}{$\begin{array}{l}\text { Insertion of } 30-\mathrm{g} \text { orthodontic force appliance } \\
\text { ( } 5 \text {-mm nickel-titanium closed-coil spring) on between } \\
\text { the left maxillary first molar and the splinted two incisors. }\end{array}$} & $\begin{array}{l}\text { The linear stage of orthodontic } \\
\text { tooth movement (M1-M2) in rats }\end{array}$ \\
\hline 3 days & 7 days & 14 days \\
\hline Measure & Measure $\uparrow$ & Measure \\
\hline \multicolumn{2}{|c|}{$\begin{array}{l}0.5 \mathrm{~mL} \text { normal saline daily for group } A \\
0.83 \mathrm{mg} / \mathrm{kg} \text { nicotine daily for group } B \\
1.67 \mathrm{mg} / \mathrm{kg} \text { nicotine daily for group C } \\
\text { administered intraperitoneal injections } \\
\text { from insertion of orthodontic appliance. }\end{array}$} & $\begin{array}{l}\text { On days } 3,7 \text {, and } 14 \text {, randomly selected rats } \\
\text { in each group were euthanized. } \\
\text { The orthodontic tooth movement rate, } \\
\text { volumetric bone changes, and osteoclasts } \\
\text { were measured using micro-computed } \\
\text { tomography and tartrate-resistant acid } \\
\text { phosphatase immunostaining. }\end{array}$ \\
\hline
\end{tabular}

Figure 1. Flowchart showing the design and timeline of the study performed to assess the effects of nicotine on orthodontic tooth movement and bone remodeling in rats. M1, first molar; M2, second molar. that was placed between the maxillary left first molar (M1) and the two splinted incisors and tied with a 0.012-inch stainless steel ligature wire, with delivery of a $30-g$ mesially directed force to the two anchored incisors (Figure 2). A dial tension gauge (DT-50; Teclock, Nagano, Japan) was used to measure the force and ensure that an identical 30-g force was applied in each animal. Because of the lack of undercuts, the lingual curvature and the eruption pattern of the two maxillary incisors, a sharp, labial-cervical, edge-shaped groove was prepared using a rotary dental disc (NTI-Kahla GmbH, Kahla, Germany) in the gingival third of both incisors in order to prevent dislodgment of the ligature wire (Figure 2B). M1 in rats has a slight bulge on the mesiopalatal surface. Accordingly, after passage of the ligature wire through the curvature under the interdental gingiva beneath the contact between $\mathrm{M} 1$ and second molar (M2), the wire was threaded under the slight bulge on the mesiopalatal surface of M1. Then, the wire end was buccally turned out in order to relieve discomfort in the palatal area. To reinforce the anterior anchorage, minimize physiological natural distal drifting of the molars, and minimize the continuous eruption of the incisors, the two incisors were joined together to act as a unit (Figure 2C). Selfetching, automix, dual-cured permanent adhesive resin cement (ZIRCONITE ${ }^{\mathrm{TM}}$, B.J.M. Laboratories Ltd., Or Yehuda, Israel) was light-cured at both ends of the ligature wire; this secured the Ni-Ti coil spring (Figure 2D). All these steps were repeatedly practiced on a pre-made cast model in order to prepare rat teeth which are 50 times smaller than that of the human molar (Figure 2A). Following insertion of the orthodontic appliance, the rats were allowed to recover with an incandescent light for warmth in their cages. All the animals were fed standard food and sufficient water ad libitum.

On days 3, 7, and 14 of OTM, randomly selected rats from each group were euthanized by carbon dioxide inhalation. The maxilla was enucleated and fixed in 10\% neutral paraformaldehyde liquid (Corefix W80; Golden Biotech. Inc., Damyang, Korea) at $4^{\circ} \mathrm{C}$ for 5 days.
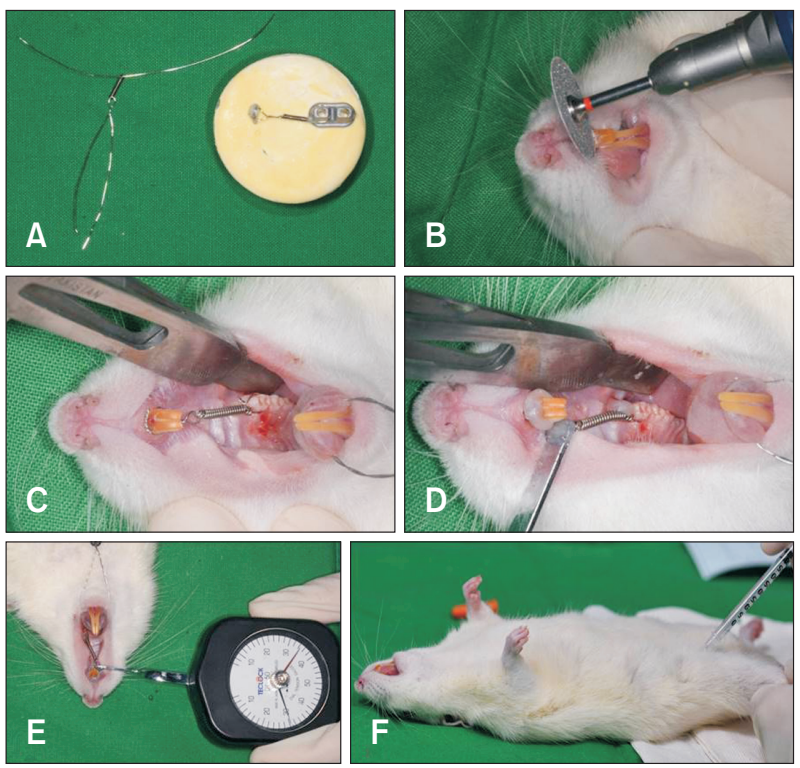

Figure 2. The 30-g orthodontic force application system used to assess the effects of nicotine on orthodontic tooth movement and bone remodeling in rats. A, A premade cast for image-based training and practice, with a 5-mm nickel-titanium ( $\mathrm{Ni}-\mathrm{Ti}$ ) closed-coil spring tied with a 0.012-inch stainless steel ligature wire and a loop for the tension gauge. B, Placement of a sharp, labialcervical, edge-shaped groove in the gingival third of the maxillary central incisors for prevention of ligature displacement. C, A 5-mm Ni-Ti closed-coil spring placed between the first molar and the two incisors, which are splinted for reinforcement of the anterior anchorage. D, Self-etching, automix, dual-cured permanent adhesive resin cement was light-cured to both ends of the ligature wire to secure the $\mathrm{Ni}-\mathrm{Ti}$ coil spring. $\mathrm{E}$, Measurement of the $30-g$ orthodontic force using a dial tension gauge. $F$, Intraperitoneal injection in the left groin area to induce anesthesia for insertion of the orthodontic appliance. 


\section{Micro-CT analyses}

For micro-CT (SkyScan 1173 ver.1.6.0; Bruker, Kontich, Belgium), the X-ray tube source voltage was set to 130 $\mathrm{kV}$, the anode electrical source current was $60 \mu \mathrm{A}$, and the image pixel size was $7.10 \mu \mathrm{m}$. Three-dimensional (3D) images were reconstructed using the micro-reconstruction NRecon software (ver. 1.7.0.4; Bruker).

\section{Linear intermolar measurements}

The 0TM rate (intermolar distance, $\mathrm{M} 1-\mathrm{M} 2, \mathrm{~mm}$ ) was defined as the distance between the most distal point on the $\mathrm{M} 1$ crown and the most mesial point on the $\mathrm{M} 2$ crown. M1-M2 was measured on two-dimensional (2D) micro-CT sections that were reoriented such that both the cementoenamel junction and the root apex of M1 and M2 appeared in the same slice. Measurements were performed in the sagittal plane, which showed the maximum root structure, at the closest proximity of the two convex molar crown surfaces. M1-M2 in each specimen of each group had been measured on three sagittal micro-CT sections before the orthodontic appliance was untied, which prevented possible errors resulting from relapse (Figure 3). The same operator repeated the measurements three times and calculated the mean value for each animal in order to enhance the intraexaminer reproducibility and reliability.

\section{Volumetric bone measurements}

Quantitative analyses of bone changes occurring in the region of $\mathrm{M} 1$ were measured in a 3D region of interest (ROI) (Figure 4), which was defined as follows: vertical, below the roof of the furcation and above the root apex; transverse, the space between the buccal and lingual cortical bone; and sagittal, 100 sections (13 $\mu \mathrm{m}$ ) beginning at the mesial root and continuing to the distal root. This 3D ROl was generated by the software based on the resultant 2D contours. The same examiner evaluated all animal specimens and used morphological landmarks when drawing ROls (CTAn 1.17.7.2+) in order to maximize bone quantification, minimize the inclusion of tooth roots, and use as many reproducible landmarks as possible (Figure $4 \mathrm{C}$ ). Volumetric bone parameters measured using the established algorithms were as follows: bone volume fraction (bone volume/total volume, $\%$ ), bone mineral density (apparent bone mineral density in the trabecular bone, $\mathrm{g} / \mathrm{cm}^{3}$ ), trabecular thickness (maximal spheres in the bone structure, $\mathrm{mm}$ ), and trabecular volume $\left(\mathrm{mm}^{3}\right)$.

\section{TRAP-positive multinucleated osteoclasts}

The samples were decalcified in 14\% ethylenediamine-tetraacetic acid for 3 weeks and subsequently processed for standard paraffin embedding. TRAP stain-

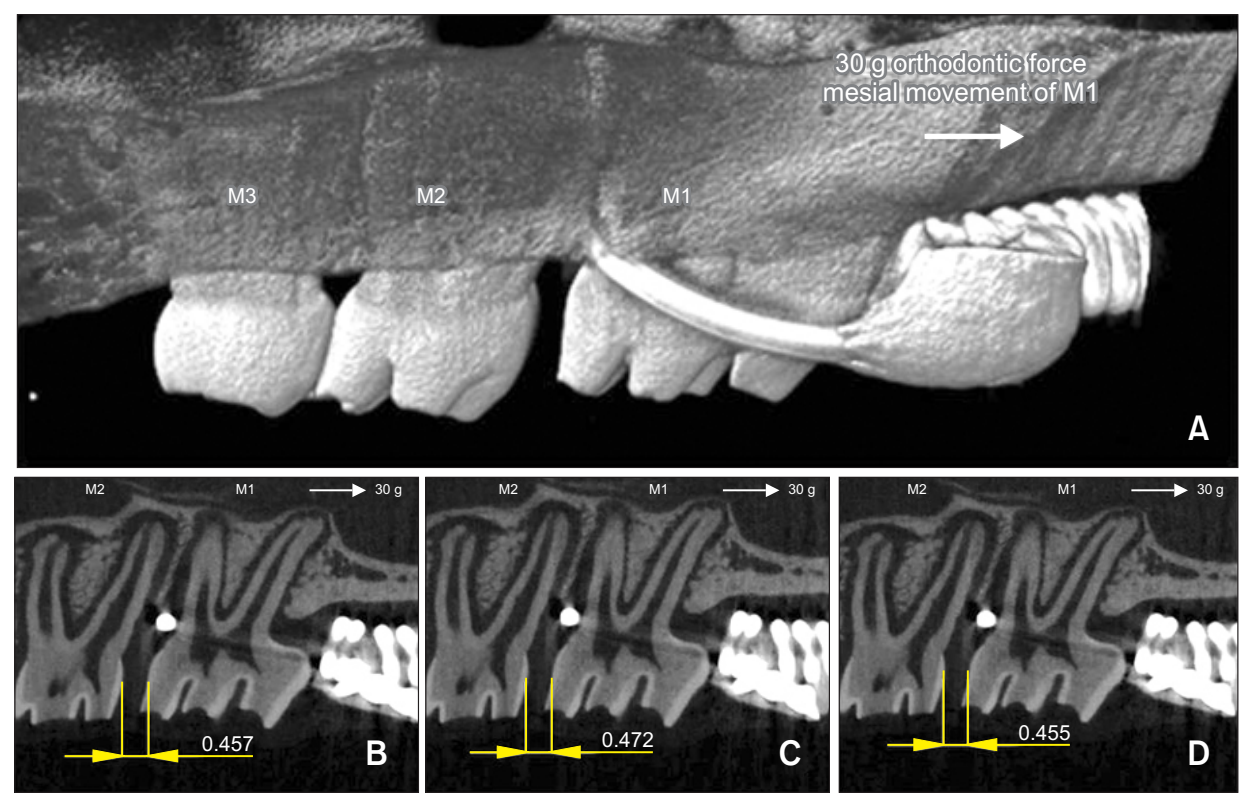

Figure 3. Micro-computed tomography (micro-CT) analyses of the effects of nicotine on orthodontic tooth movement and bone remodeling in rats. $A$, Linear measurements. The intermolar distance (M1-M2, $\mathrm{mm}$ ) had been measured on three sagittal micro-CT sections for each animal in each group before the orthodontic appliance was untied, which prevented possible errors resulting from relapse. B-D, M1-M2 $(\mathrm{mm})$ was measured on three bidimensional micro-CT sections that were reoriented such that both the cementoenamel junction and the root apex of M1-M2 appeared in the same slice. The same operator repeated the measurements three times and calculated the mean value for each specimen. M1, first molar; M2, second molar; M3, third molar. 

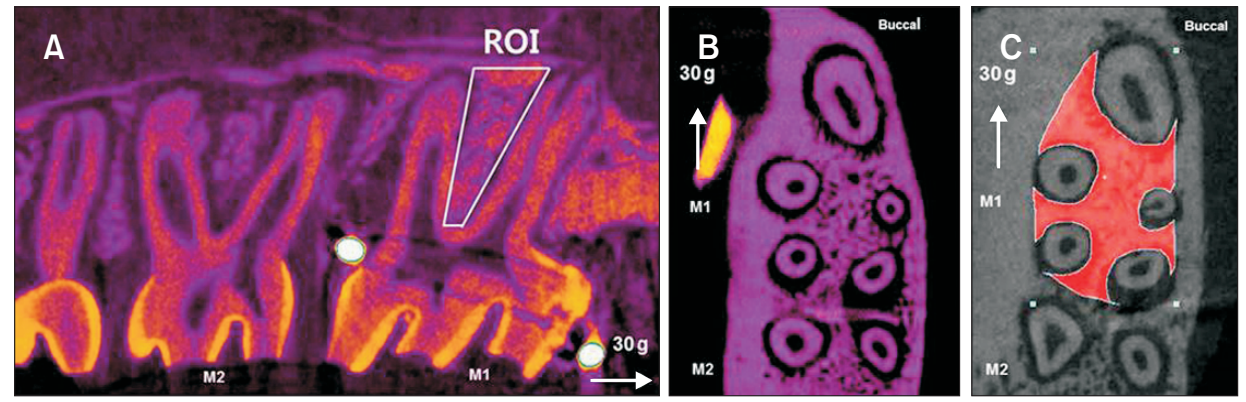

Figure 4. Volumetric measurements obtained by micro-computed tomography to assess the effects of nicotine on orthodontic tooth movement and bone remodeling in rats. A, A three-dimensional region of interest (ROI) was marked for quantitative analyses of bone changes occurring in the region of the maxillary first molar (M1). A, B, ROI was defined as follows: vertical, below the roof of the furcation and above the root apex; transverse, the space between the buccal and lingual cortical bone; and sagittal, 100 sections $(13 \mu \mathrm{m})$ beginning at the mesial root and continuing to distal root. C, The same examiner evaluated all animal specimens and used morphological landmarks when drawing ROls (CTAn 1.17.7.2+) in order to maximize bone quantification, minimize the inclusion of tooth roots, and use as many reproducible landmarks as possible.

M1, first molar; M2, second molar.

ing was performed using polymerase chain reaction (PCR)-Cy5 fluorescent gel-based telomerase repeated amplification protocol. TRAP-positive osteoclasts were counted on the mesial alveolar bone surface of the distobuccal root of M1, defined as the compression side during OTM (Figure 5). ${ }^{11,18}$ The area for quantification included a square with one side extending from the root apex to the bifurcation and the other side extending $200 \mu \mathrm{m}$ from the border of the periodontal ligament inside the alveolar bone. ${ }^{11}$ Digital pathology software (CaseViewer 2.2.1; 3DHISTECH, Budapest, Hungary) was used on the identically magnifying sectioned image by expressing annotations on each TRAP-positive multinucleated osteoclast (Figure 5B).

\section{Statistical analysis}

Six dependent outcome variables, including the 1) intermolar distance, 2) bone volume fraction, 3) bone mineral density, 4) trabecular thickness, 5) trabecular volume, and 6) osteoclast number, were summarized using simple descriptive statistics. Because of the sample size, the nonparametric Kruskal-Wallis test was performed using SPSS ver. 23.0 (IBM Corp., Armonk, NY, USA) to evaluate statistically significant differences among groups at 3, 7, and 14 days of OTM (reflecting the dose- and time-dependent effects of nicotine). A $p$ value of $<0.05$ was considered statistically significant.

Taking into account ethical considerations, the high cost of micro-CT analyses, and the degree of statistical certainty, it was necessary to recruit a minimum number of experimental animals. In most instances, nonparametric tests are slightly less efficient even when the assumptions of normality, independence within and

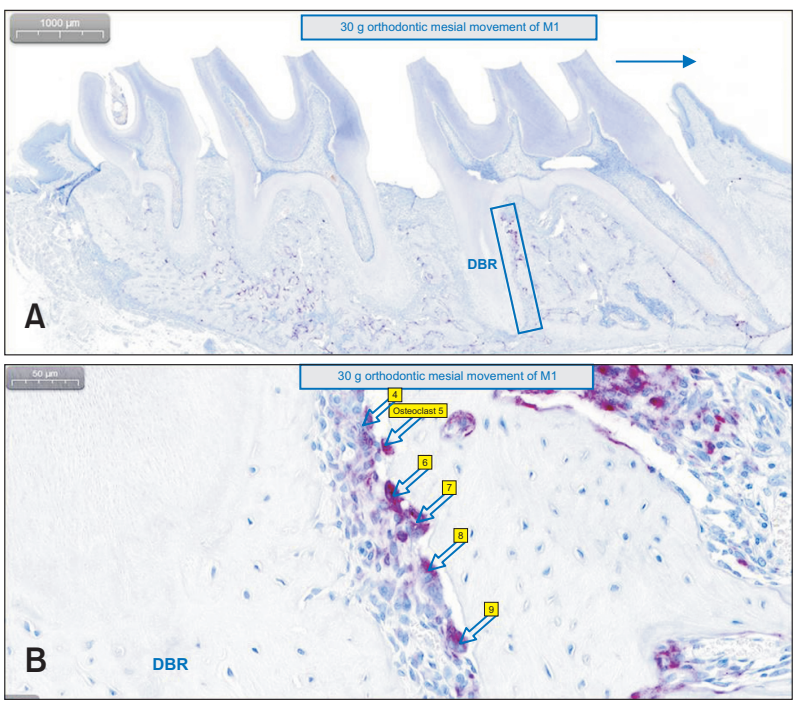

Figure 5. Counting of tartrate-resistant acid phosphatase (TRAP)-positive multinucleated osteoclasts on the mesial alveolar bone surface of the distobuccal root (DBR) of the maxillary left first molar (M1), to assess the effects of nicotine on orthodontic tooth movement and bone remodeling in rats. Digital pathology software was used on identically magnifying sectioned images. A, The area for quantification (blue rectangular block on the image) includes a square with one side extending from the root apex to the bifurcation and the other side extending 200 $\mu \mathrm{m}$ from the border of the periodontal ligament inside the alveolar bone of DBR of M1. 1,000 $\mu \mathrm{m}$ (original image $\times 10)$. B, The arrows indicate each TRAP-positive multinucleated osteoclast with expressed annotations by the software. $50 \mu \mathrm{m}$ (original image $\times 300$ ). 
between samples, identical distribution of each element, and equal variances of the samples are satisfied. On the other hand, they can be significantly more efficient (i.e., require a much smaller sample size) when these assumptions are not satisfied. ${ }^{15,16}$

Spearman's correlation analyses were conducted to investigate the relationship of the dependent outcome variables with the OTM rate in the three groups at 3, 7, 14 days.

\section{RESULTS}

\section{Participants}

From the 48 animals that were originally fed and prepared for the study, nine were excluded; four could not be awakened from general anesthesia administered for orthodontic appliance insertion; two died after nicotine injection on the fourth and eleventh days of OTM, respectively; and three were euthanized after dislodgment of the orthodontic appliance. Thus, 39 adult male rats were included in the final analysis. There were nine rats in control group of A ( 3 per 3, 7, and 14 days) and fifteen rats each in experimental groups of B and C ( 5 per 3 , 7 , and 14 days).

\section{Linear intermolar measurements}

The intermolar distance (M1-M2, mm) showed no statistically significant difference among groups at 3, 7 , and 14 days. However, the maximum distance was observed on day 14 in group $C$, while the least distance was observed on day 3 in group A (Figure 6). According to the descriptive statistics, the OTM rate in the control group gradually increased at regular intervals over time. Compared with the control group, the nicotine groups showed a fluctuating OTM rate over the observation period (Figure $7 \mathrm{~A}$ ).

\section{Volumetric bone measurements}

The bone volume fraction, bone mineral density, trabecular thickness, and trabecular volume showed no statistically significant differences among groups at 3, 7 , and 14 days. However, the lowest values for the bone volume fraction, bone mineral density, and trabecular thickness were observed on day 14 in group C (Figure 8). Spearman's correlation analyses showed a significant relationship $(p<0.01)$ between $\mathrm{M} 1-\mathrm{M} 2$ and the bone volume fraction, bone mineral density, and trabecular thickness on day 7 in group A and day 14 in group C.
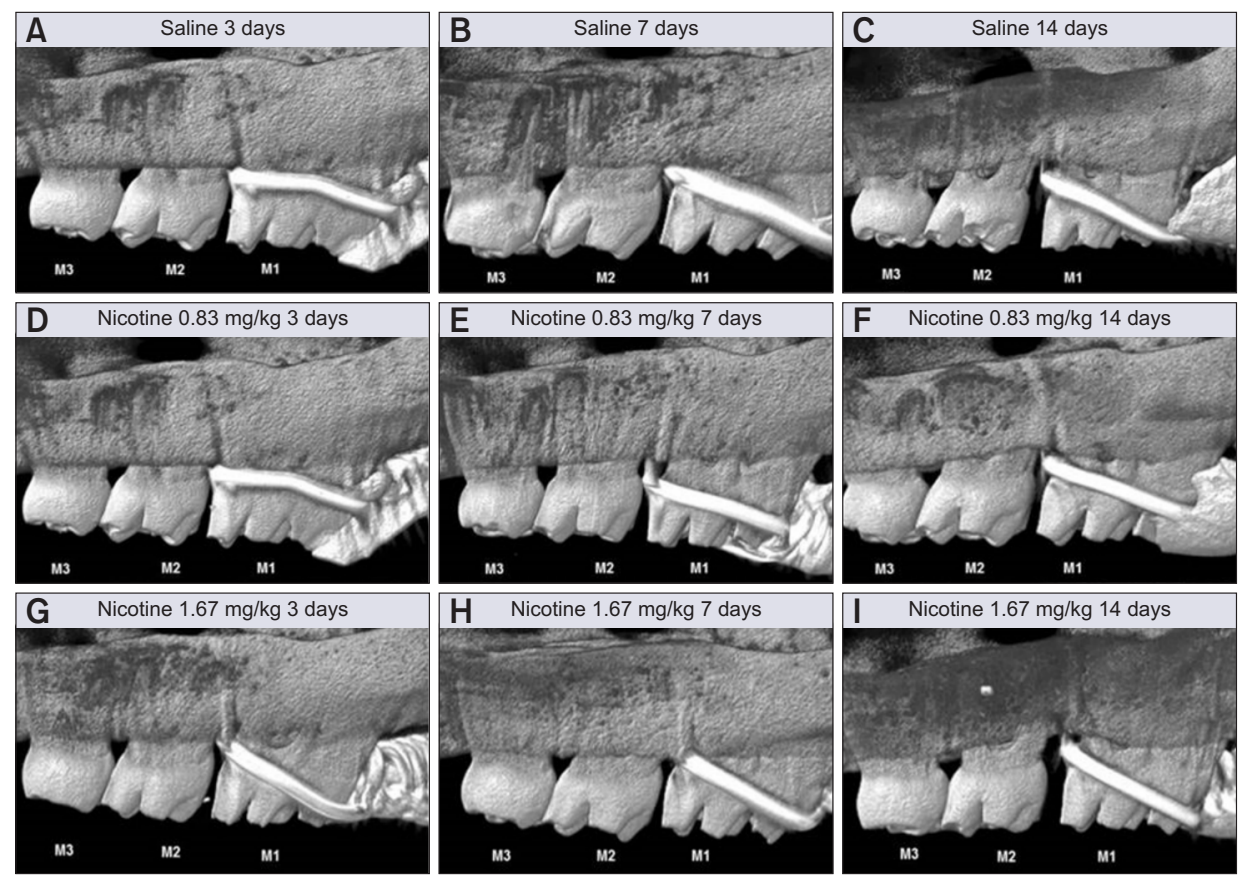

Figure 6. Linear intermolar measurements obtained to assess the effects of nicotine on orthodontic tooth movement and bone remodeling in rats. Representative images for each group showing the intermolar distance (amount of orthodontic tooth movement, $\mathrm{M} 1-\mathrm{M} 2, \mathrm{~mm}$ ) on the left side of maxilla at 3, 7, and 14 days after insertion of a 30-g orthodontic force application device (image magnification $\times 10$ ). A-C, Control group (saline $0.5 \mathrm{~mL}$ daily). D-F, Experimental group (nicotine $0.83 \mathrm{mg} / \mathrm{kg}$ daily). G-I, Experimental group (nicotine $1.67 \mathrm{mg} / \mathrm{kg}$ daily).

M1, first molar; M2, second molar; M3, third molar. 
A

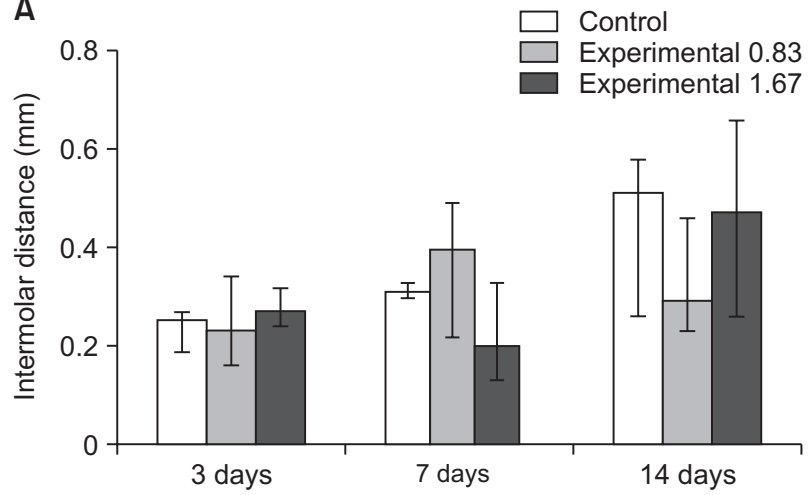

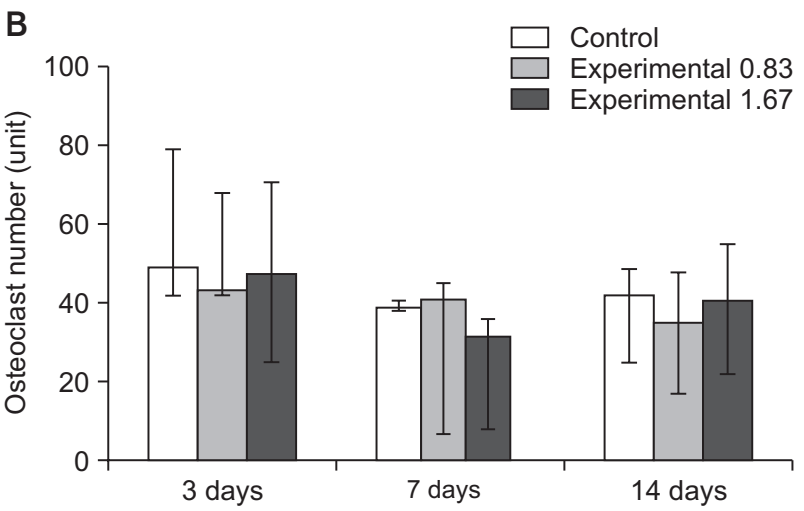

Figure 7. Comparative observations of the intermolar distance and osteoclast number at 3, 7, and 14 days after insertion of a 30-g orthodontic force application device in the control (saline $0.5 \mathrm{~mL}$ daily, white bar) and experimental (nicotine $0.83 \mathrm{mg} / \mathrm{kg}$ daily, grey bar; nicotine $1.67 \mathrm{mg} / \mathrm{kg}$ daily, deep gray bar) groups, to assess the effects of nicotine on orthodontic tooth movement and bone remodeling in rats. A, Intermolar distance. B, Osteoclast number. The median, maximum, and minimum values at 3,7 , and 14 days were expressed as the representative values for each group.
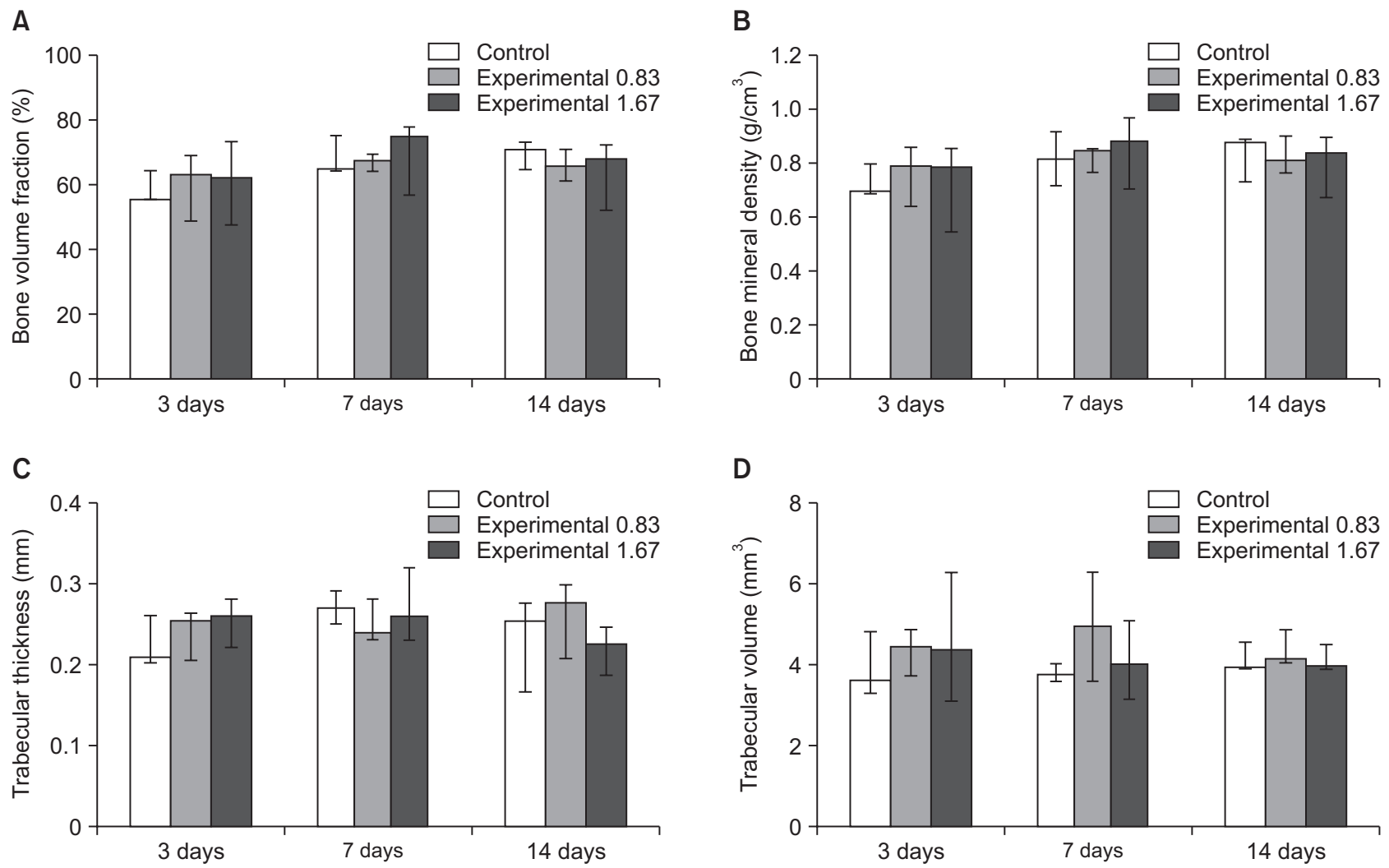

Figure 8. Volumetric measurements of trabecular bone changes occurring in the region of interest in the left maxillary first molar at 3, 7, and 14 days after insertion of a 30-g orthodontic force application device of in the control (saline $0.5 \mathrm{~mL}$ daily, white bar) and experimental (nicotine $0.83 \mathrm{mg} / \mathrm{kg}$ daily, grey bar; nicotine $1.67 \mathrm{mg} / \mathrm{kg}$ daily, deep gray bar) groups, to assess the effects of nicotine on orthodontic tooth movement and bone remodeling in rats. $A$, Bone volume fraction. B, Bone mineral density. C, Trabecular thickness. D, Trabecular volume. The median, maximum, and minimum values at 3,7 , and 14 days were expressed as the representative values for each group. 

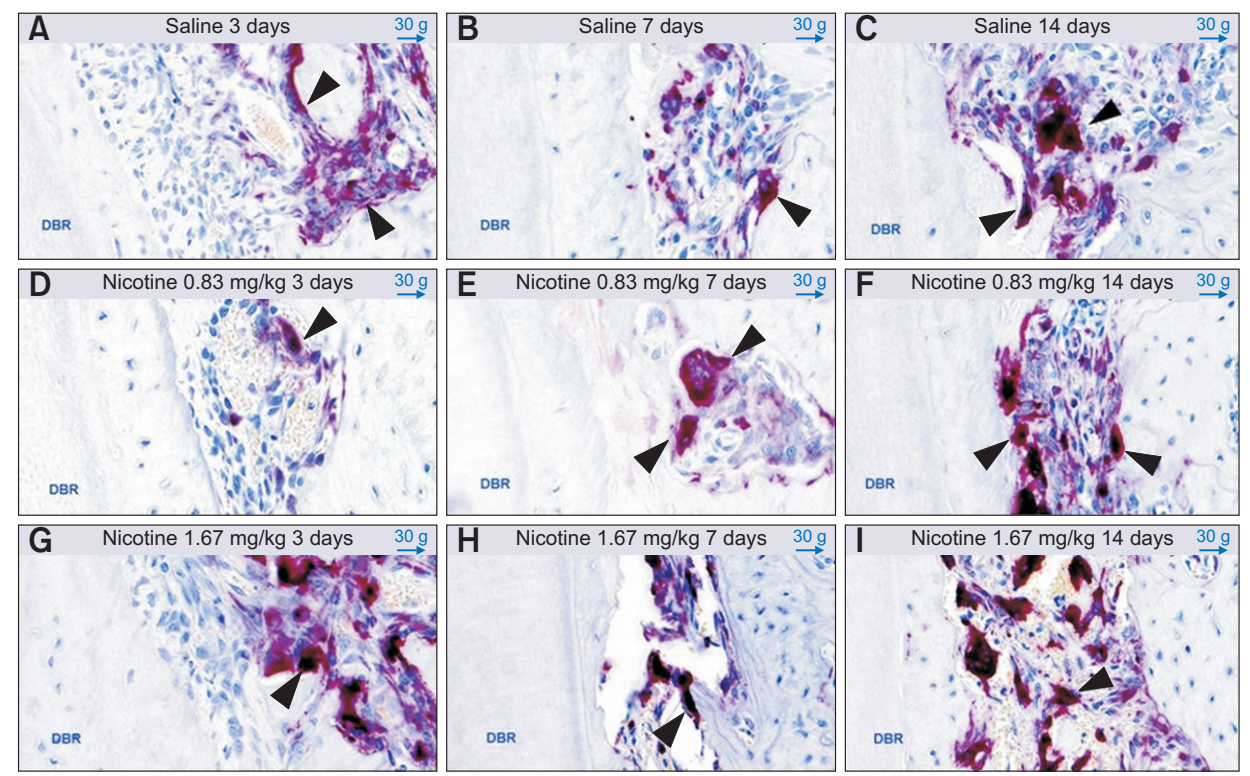

Figure 9. Evaluation of tartrate-resistant acid phosphatase-positive multinucleated osteoclasts (black arrows) on the mesial alveolar bone surface of the distobuccal root (DBR) of the maxillary left first molar at 3, 7, and 14 days after insertion of a 30-g orthodontic force application device to assess the effects of nicotine on orthodontic tooth movement and bone remodeling in rats. Representative images $(50-\mu \mathrm{m}$, original image $\times 300)$ for each group are shown. A-C, Control group (saline $0.5 \mathrm{~mL}$ daily). D-F, Experimental group (nicotine $0.83 \mathrm{mg} / \mathrm{kg}$ daily). G-I, Experimental group (nicotine $1.67 \mathrm{mg} / \mathrm{kg}$ daily).

\section{TRAP-positive multinucleated osteoclasts}

The number of TRAP-positive osteoclasts showed no statistically significant differences among groups at 3 , 7, and 14 days (Figure 9). On day 14 in group C, the number of osteoclasts showed a tendency to increase; this coincided with the maximum intermolar distance observed among the three groups and three time points (Figure 7B).

\section{DISCUSSION}

The alveolar bone in rats is generally more dense than that in humans ${ }^{19}$ and tissue changes in rats during orthodontic treatment appear to be faster than those in humans, although the principal mechanisms are the same. ${ }^{20}$ Despite these differences, rats are generally considered a good model for well-defined, standardized, reproducible force delivery system. ${ }^{21}$ Tissue damage and remodeling in rats start within a few hours, and studies aimed at describing the biological response in the linear phase of tooth movement should have an experimental period of at least 2 weeks (14 days). ${ }^{8,19}$

One of the major concerns related to experimental animal studies is the possibility of extrapolating the findings to human clinical settings. According to a recent (2018) systematic review of experimental studies in rats, ${ }^{22}$ nicotine exposure in rats jeopardizes OTM by in- creasing alveolar bone loss and root resorption. The authors also concluded that further studies need to assess the impact of habitual use of tobacco products on OTM from a clinical perspective. The present study sought to investigate the effect of nicotine on the initial, lag, and post-lag stages of OTM and determine the stage at which the consequences of nicotine exposure and orthodontically-induced inflammation were observed.

Shintcovsk et al. ${ }^{14}$ showed significant differences in the effect of nicotine on bone remodeling at 3, 7, and 14 days of OTM in rats. Nociti Jr et al. ${ }^{17}$ revealed that daily administration of nicotine $0.37 \mathrm{mg} / \mathrm{kg}, 0.57 \mathrm{mg} /$ $\mathrm{kg}$, or $0.73 \mathrm{mg} / \mathrm{kg}$ could produce direct deleterious effects on the periodontal tissues in rats, including those without periodontitis. The direct negative effects of the two doses of nicotine $0.83 \mathrm{mg} / \mathrm{kg}$ daily and $1.67 \mathrm{mg} / \mathrm{kg}$ daily selected in the present study, and orthodonticallyinduced inflammation could start within a few hours in rats. ${ }^{17}$ Liu et al. ${ }^{6}$ performed micro-CT analysis and showed that nicotine injection after ligation (= induction of periodontitis) for 14 and 28 days increased further alveolar bone loss and decreased the bone mineral density, bone volume fraction, and trabecular thickness in a dose-dependent manner $(p<0.05)$. They also found that in the absence of periodontitis, nicotine itself caused alveolar bone loss in a dose-dependent manner.

In the present study, 5-mm Ni-Ti closed-coil springs 
were used to produce a uniform and continuous 30-g orthodontic force during OTM. ${ }^{23}$ King et al. ${ }^{23}$ demonstrated that effective movement of rat molars required a force of 20 to $40 \mathrm{~g}$, and that the velocity of tooth movement did not increase at a force of $>40 \mathrm{~g}$. In a previous study, intermolar distance between $\mathrm{M} 1$ and $\mathrm{M} 2$ at the linear stage of OTM were reported about 0.28 $\mathrm{mm} /$ week in $20 \mathrm{~g}$ force, $0.16 \mathrm{~mm} /$ week in $40 \mathrm{~g}$, and 0.17 $\mathrm{mm} /$ week in $60 \mathrm{~g}$ for 14 days. ${ }^{23}$ Sodagar et al. ${ }^{12}$ performed an in vivo study and showed that nicotine significantly accelerated the OTM rate in a dose-dependent manner. Accordingly, they suggested that orthodontists should advise their patients to use nicotine replacement therapies to aid in smoking cessation. However, the authors measured M1-M2 (mm) using an interproximal gauge only at 14 days without any other measurement methods. ${ }^{12}$ Bakathir et al. ${ }^{13}$ found that nicotine significantly accelerated the OTM rate in a dose-dependent manner; this resulted from unbalanced bone resorption and apposition around moving teeth in rats, as observed through histological assessments. The authors highlighted multiple complications that smokers might experience during and after OTM. ${ }^{13}$ However, they also measured M1-M2 ( $\mathrm{mm})$ using an interproximal gauge only at 14 days. On the other hand, in the present study, a single examiner measured M1-M2 (mm) at 3, 7, and 14 days in accordance with the stages of OTM on 2D sagittal micro-CT sections. In agreement with the abovementioned previous studies, ${ }^{12,13}$ the maximum intermolar distance was observed on day 14 in the group receiving the highest dose of nicotine, although the difference among groups was not statistically significant.

High-resolution micro-CT analyses measure bone parameters and provide detailed, highly reliable, accurate and reproducible 3D information about alveolar hard tissue changes over time in a precise and noninvasive manner. ${ }^{6,24}$ In the present study, the fluctuating hard tissue changes over the observation period in the nicotine groups might be associated with the biphasic effects of nicotine on both osteoblasts and osteoclasts. The effects of nicotine on cell proliferation are biphasic; with toxic, antiproliferative effects at high doses ( $>1 \mathrm{mmol} /$ $\mathrm{L})$ and stimulatory effects at very low doses (0.01-10 $\mu \mathrm{mol} / \mathrm{L}) .{ }^{25,26}$ The trabecular bone changes observed in the present study showed no significant among groupdifferences, although they were similar to the changes observed through micro-CT analyses by liu et al. ${ }^{6}$ Moreover, our Spearman's correlation analyses showed a significant relationship $(p<0.01)$ between M1-M2 and the bone volume fraction, bone mineral density, and trabecular thickness on day 7 in group A and day 14 in group C.

The correlation between the number of osteoclasts and the time- and dose-dependent effects of nicotine during the different stages of OTM has not been widely investigated, with controversial results published in the literature. In the present study, the number of TRAPpositive osteoclasts tended to increase on day 14 in group C, where the nicotine dose was $1.67 \mathrm{mg} / \mathrm{kg}$; this coincided with the finding of the maximum intermolar distance among the three groups and three time points.

Shintcovsk et al. ${ }^{14}$ reported that male rats who received $2 \mathrm{mg} / \mathrm{kg}$ nicotine showed significantly fewer osteoclast-like cells at 7 and 14 days of OTM. Kirschneck et al. $^{27}$ demonstrated that orthodontic force application in vivo led to a significant increase in nicotine-induced periodontal bone loss, likewise force application in vitro enhanced the differentiation of RAW264.7 precursor cells into osteoclast-like cells. For the in vivo study, they only used one dose of nicotine $1.89 \mathrm{mg} / \mathrm{kg}$ and a small sample of 14 male rats. Araujo et al. ${ }^{28}$ reported that nicotine decreased the number of osteoclasts when dental movement was not induced (nicotine $1 \mathrm{mg} / \mathrm{kg}$ without tooth movement group). They calculated the mean number of osteoclasts per square micrometer of all periodontal ligaments in order to avoid the risk of bias related to selection of the compression side of the periodontal ligament and alveolar bone. ${ }^{28}$ Gonzales et al. ${ }^{18}$ measured M1-M2 using digitized lateral cephalograms and observed the largest and deepest root resorption craters on the disto-buccal root which appeared to the compression zone, using 3D images obtained via a laser scanning electron microscope. In the present study, osteoclasts were counted on the mesial alveolar bone surface of the distobuccal root of M1, which was defined as the compression side of OTM. ${ }^{11}$

In addition, nicotine has been reported to induce cytotoxicity and affect bone remodeling through downregulation of osteoprotegerin expression and upregulation of receptor activator of nuclear factor-kappa B ligand expression in periodontal ligament cells. ${ }^{29}$ Recently, ferric nitrilotriacetate was found to be an oxidizing agent that affects bone metabolism by suppressing bone growth and increasing cytokines as interleukin (IL)-1 and IL-6, involved in bone resorption. ${ }^{30}$

\section{CONCLUSION}

The findings of this study suggest that nicotine does not affect OTM and bone remodeling, although fluctuations according to the stage of OTM in the nicotine groups should be clearly elucidated in further prospective studies. Currently, it is necessary to accumulate more profound scientific evidence and knowledge regarding the effects of nicotine on OTM and bone remodeling.

Based on available evidence, orthodontists should explain critical guidelines and precautions to current smokers before the initiation of any orthodontic treat- 
ment. With implementation of the Plan-Do-Study-Act protocol, future studies with different methods and strategies to overcome the limitations of the present study such as recruitment of more samples, observation over a longer period, and utilization of the advantages of micro-CT without compromises to obtain pharmacokinetic data from potentially fewer animals, will further clarify our findings.

\section{CONFLICTS OF INTEREST}

No potential conflict of interest relevant to this article was reported.

\section{REFERENCES}

1. César-Neto JB, Duarte PM, Sallum EA, Barbieri D, Moreno $\mathrm{H}$ Jr, Nociti FH Jr. A comparative study on the effect of nicotine administration and cigarette smoke inhalation on bone healing around titanium implants. J Periodontol 2003;74:1454-9.

2. Hapidin H, Othman F, Soelaiman IN, Shuid AN, Luke DA, Mohamed N. Negative effects of nicotine on bone-resorbing cytokines and bone histomorphometric parameters in male rats. J Bone Miner Metab 2007;25:93-8.

3. Suda N, Morita 1, Kuroda T, Murota S. Participation of oxidative stress in the process of osteoclast differentiation. Biochim Biophys Acta 1993;1157:31823.

4. Mody N, Parhami F, Sarafian TA, Demer LL. Oxidative stress modulates osteoblastic differentiation of vascular and bone cells. Free Radic Biol Med 2001;31:509-19.

5. Bosco AF, Bonfante S, de Almeida JM, Luize DS, Nagata MJ, Garcia VG. A histologic and histometric assessment of the influence of nicotine on alveolar bone loss in rats. J Periodontol 2007;78:527-32.

6. Liu YF, Wu LA, Wang J, Wen LY, Wang XJ. Microcomputerized tomography analysis of alveolar bone loss in ligature- and nicotine-induced experimental periodontitis in rats. J Periodontal Res 2010;45:7149.

7. César-Neto JB, Benatti BB, Sallum EA, Casati MZ, Nociti FH Jr. The influence of cigarette smoke inhalation and its cessation on the tooth-supporting alveolar bone: a histometric study in rats. J Periodontal Res 2006;41:118-23.

8. Krishnan V, Davidovitch Z. Cellular, molecular, and tissue-level reactions to orthodontic force. Am J Orthod Dentofacial Orthop 2006;129:469.e1-32.

9. Gameiro GH, Pereira-Neto JS, Magnani MB, Nouer DF. The influence of drugs and systemic factors on orthodontic tooth movement. J Clin Orthod
2007;41:73-8; quiz 71.

10. lino S, Sakoda S, Ito G, Nishimori T, Ikeda T, Miyawaki S. Acceleration of orthodontic tooth movement by alveolar corticotomy in the dog. Am J Orthod Dentofacial Orthop 2007;131:448.e1-8.

11. Yadav S, Dobie T, Assefnia A, Gupta H, Kalajzic Z, Nanda R. Effect of low-frequency mechanical vibration on orthodontic tooth movement. Am J Orthod Dentofacial Orthop 2015;148:440-9.

12. Sodagar A, Donyavi Z, Arab S, Kharrazifard MJ. Effect of nicotine on orthodontic tooth movement in rats. Am J Orthod Dentofacial Orthop 2011;139: e261-5.

13. Bakathir MA, Linjawi $\mathrm{Al}$, Omar SS, Aboqura $\mathrm{AB}$, Hassan AH. Effects of nicotine on bone during orthodontic tooth movement in male rats. Histological and immunohistochemical study. Saudi Med J 2016; 37:1127-35.

14. Shintcovsk RL, Knop L, Tanaka OM, Maruo H. Nicotine effect on bone remodeling during orthodontic tooth movement: histological study in rats. Dental Press J Orthod 2014;19:96-107.

15. Eckelman WC, Kilbourn MR, Joyal JL, Labiris R, Valliant JF. Justifying the number of animals for each experiment. Nucl Med Biol 2007;34:229-32.

16. Scheibe PO. Number of samples - hypothesis testing. Nucl Med Biol 2008;35:3-9.

17. Nociti FH Jr, Nogueira-Filho GR, Tramontina VA, Machado MA, Barros SP, Sallum EA, et al. Histometric evaluation of the effect of nicotine administration on periodontal breakdown: an in vivo study. J Periodontal Res 2001;36:361-6.

18. Gonzales C, Hotokezaka H, Yoshimatsu M, Yozgatian JH, Darendeliler MA, Yoshida N. Force magnitude and duration effects on amount of tooth movement and root resorption in the rat molar. Angle Orthod 2008;78:502-9.

19. Reitan K, Kvam E. Comparative behavior of human and animal tissue during experimental tooth movement. Angle Orthod 1971;41:1-14.

20. Brudvik P, Rygh P. The initial phase of orthodontic root resorption incident to local compression of the periodontal ligament. Eur J Orthod 1993;15:24963.

21. Ren Y, Maltha JC, Kuijpers-Jagtman AM. The rat as a model for orthodontic tooth movement--a critical review and a proposed solution. Eur J Orthod 2004;26:483-90.

22. Michelogiannakis D, Rossouw PE, Al-Shammery D, Akram Z, Khan J, Romanos GE, et al. Influence of nicotine on orthodontic tooth movement: a systematic review of experimental studies in rats. Arch Oral Biol 2018;93:66-73.

23. King GJ, Keeling SD, McCoy EA, Ward TH. Measur- 
ing dental drift and orthodontic tooth movement in response to various initial forces in adult rats. Am J Orthod Dentofacial Orthop 1991;99:456-65.

24. Bouxsein ML, Boyd SK, Christiansen BA, Guldberg RE, Jepsen KJ, Müller R. Guidelines for assessment of bone microstructure in rodents using micro-computed tomography. J Bone Miner Res 2010;25:146886.

25. Walker LM, Preston MR, Magnay JL, Thomas PB, El Haj AJ. Nicotinic regulation of c-fos and osteopontin expression in human-derived osteoblast-like cells and human trabecular bone organ culture. Bone 2001;28:603-8.

26. Henemyre CL, Scales DK, Hokett SD, Cuenin MF, Peacock ME, Parker MH, et al. Nicotine stimulates osteoclast resorption in a porcine marrow cell model. J Periodontol 2003;74:1440-6.
27. Kirschneck C, Proff P, Maurer M, Reicheneder C, Römer P. Orthodontic forces add to nicotineinduced loss of periodontal bone: an in vivo and in vitro study. J Orofac Orthop 2015;76:195-212.

28. Araujo CM, Rocha AC, Araujo BMM, Johann ACBR, Pereira LF, Tanaka OM, et al. Effect of acute administration of nicotine and ethanol on tooth movement in rats. Braz Oral Res 2018;32:e96.

29. Lee HJ, Pi SH, Kim Y, Kim HS, Kim SJ, Kim YS, et al. Effects of nicotine on antioxidant defense enzymes and RANKL expression in human periodontal ligament cells. J Periodontol 2009;80:1281-8.

30. Norazlina M, Hermizi H, Faizah O, Nazrun AS, Norliza M, Ima-Nirwana S. Vitamin E reversed nicotineinduced toxic effects on bone biochemical markers in male rats. Arch Med Sci 2010;6:505-12. 